\title{
Vaccin contre le SRAS-CoV-2 : défis et considérations
}

\author{
Ruchi Chaube ${ }^{1 *}$
}

\section{Résumé}

Il est essentiel de tenir compte des défis auxquels nous avons déjà été confrontés et auxquels nous avons répondu dans le cadre de la mise au point d'un vaccin contre le coronavirus du syndrome respiratoire aigu sévère 2 (SRAS-CoV-2). Compte tenu de la gravité de la crise sanitaire que le SRAS-CoV-2 a causée à l'échelle mondiale, et compte tenu du fait que nous en savons très peu sur le virus, nous devrions nous concentrer sur des approches qui peuvent améliorer les résultats en matière de développement dans un laps de temps relativement court. Ce commentaire traite de l'utilisation des vaccins à base d'acide nucléique (acide désoxyribonucléique et acide ribonucléique) contre les infections virales et les contextes de pandémie. Les avantages potentiels des vaccins à base d'acide nucléique par rapport aux vaccins conventionnels sont présentés, puis nous discuterons des vaccins à base d'acide nucléique en développement contre les infections virales ainsi que des défis relatifs à ces vaccins au début des essais cliniques.
Cette oeuvre est mise à la disposition selon les termes de la licence internationale Creative Commons Attribution 4.0

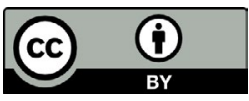

Affiliation

${ }^{1}$ Direction générale des opérations réglementaires et de l'application de la loi, Santé Canada, Edmonton, AB

*Correspondance :

ruchi.chaube@canada.ca

Citation proposée : Chaube R. Vaccin contre le SRAS-CoV-2 : défis et considérations. Relevé des maladies transmissibles au Canada 2021;47(3):141-4. https://doi.org/10.14745/ccdr.v47i03a01f

Mots-clés : vaccin, SRAS-CoV-2, acide nucléique

\section{Introduction}

Un nouveau coronavirus, le coronavirus du syndrome respiratoire aigu sévère 2 (SRAS-CoV-2), causant la maladie du coronavirus 2019 (COVID-19), a été introduit dans la population humaine et s'est rapidement répandu dans le monde au début de 2020, causant une pandémie mondiale. Cette pandémie, tel que défini par l'Organisation mondiale de la Santé, est « une épidémie qui sévit dans le monde entier, ou sur une très vaste zone, traversant les frontières internationales et touchant habituellement un grand nombre de personnes » (1), a amené les milieux scientifiques et médicaux à entreprendre de sérieux efforts pour limiter la vague de propagation virale en mettant au point des vaccins préventifs.

Un vaccin (ou plusieurs) contre le SRAS-CoV-2 aiderait à développer l'immunité communautaire contre le virus et ainsi prévenir la propagation et la réapparition de la maladie au niveau de la population. II y a eu une augmentation du nombre de vaccins-candidats depuis le début de la pandémie; toutefois, la protection contre le SRAS-CoV-2 repose sur deux questions, à savoir, premièrement, à quel moment un vaccin peut être disponible et, deuxièmement, si les vaccins offriront une protection suffisante pour empêcher complètement la propagation du virus. Bien que le premier point soit temporel et que, à l'heure actuelle, nous sommes très en avance en ce qui concerne les vaccins contre la COVID-19, le deuxième point est fondamental pour le développement d'un vaccin définissant une réponse immunologique forte et durable.
Au cours des dernières décennies, il y a eu une propagation rapide de nombreuses infections virales graves, y compris le virus de l'immunodéficience humaine $(\mathrm{VIH})$, I'influenza $A$, le syndrome respiratoire aigu sévère (SRAS), le virus Ebola et le virus Zika. Ces infections ont nécessité le développement rapide et la distribution complète de vaccins, mais le développement de ces vaccins s'est révélé extrêmement difficile. De plus, plusieurs de ces virus représentent des zoonoses (maladies zoonotiques), ce qui augmente le risque d'introduire un virus aux propriétés immunogéniques complètement nouvelles dans la population humaine. Il est également impossible de prédire les caractéristiques de ces virus, la gravité des maladies qu'ils peuvent provoquer et l'ampleur des éclosions qu'ils peuvent causer. Par exemple, le virus de l'influenza A/H1N1 a mené à une alerte pandémique de phase 6 en 2009, mais il a causé des symptômes relativement légers comparativement à la pandémie de 1918 (la « grippe espagnole »), qui a entraîné la mort de 50 millions de personnes (2).

\section{Vaccins}

\section{Vaccins conventionnels}

Les vaccins conventionnels - vivants atténués ou inactivés - se sont révélés bénéfiques contre plusieurs maladies infectieuses par le passé. Toutefois, ils ne conviennent pas toujours dans les situations d'éclosion, car ils présentent un risque de recul 
et peuvent causer des effets indésirables graves, ce qui rend cette approche défavorable pour les organismes hautement pathogènes. Ce recul a été observé avec le vaccin contre le virus Ebola (3). De plus, les vaccins conventionnels posent des défis en matière de production commerciale, car ils nécessitent la culture et la propagation de pathogènes entiers, ce qui exige l'utilisation de laboratoires de biosécurité.

Comme ces virus sont en grande partie non caractérisés avant qu'une éclosion ne se produise, le temps devient un facteur crucial pour la mise au point d'un vaccin efficace. À l'heure actuelle, le délai moyen de développement des vaccins conventionnels à partir du stade préclinique est de plus de 10 ans (4), ce qui souligne le besoin urgent d'explorer des méthodes qui permettent un développement rapide pour empêcher qu'une éclosion émergente ne devienne une pandémie.

\section{Vaccins à base de vecteur viral}

Une solution de rechange précieuse à un vaccin conventionnel est un vaccin à base de vecteur viral, car cette technique représente une plateforme très polyvalente. Les vecteurs viraux peuvent être exploités pour encoder les antigènes hétérologues qui peuvent être introduits dans les cellules hôtes. À l'intérieur de l'hôte, ils expriment les antigènes codés, incitant l'hôte à induire une réponse immunitaire. Cette plateforme semblait efficace contre le virus Ebola, et le rVSV ZEROV représente actuellement le candidat le plus prometteur pour un vaccin homologué (5). Toutefois, les vecteurs viraux ne sont pas largement utilisés, car ils sont considérés comme des risques pour la santé humaine et l'environnement étant donné qu'ils sont des organismes génétiquement modifiés. De plus, ces vecteurs présentent toujours un risque d'intégration dans le génome de l'hôte, et une réplication trop élevée ou persistante dans I'hôte soulève des préoccupations quant à leur utilisation chez I'homme (6).

\section{Vaccins $d$ 'acide nucléique}

Les vaccins d'acide nucléique, à la fois l'acide désoxyribonucléique (ADN) et l'acide ribonucléique (ARN), présentent des avantages potentiels par rapport aux vaccins conventionnels, car ils sont plus stables, plus rentables, faciles à fabriquer et à manipuler, fournissent une immunité à large spectre (ce qui signifie qu'un vaccin à plusieurs antigènes peut être conçu pour cibler efficacement des souches de virus en constante évolution) et peuvent induire des réponses immunitaires humorales et cellulaires $(7,8)$. Les vaccins d'acide nucléique ont également un avantage par rapport aux vaccins à base de vecteurs viraux, car ils sont dérivés de plasmides recombinants d'origine bactérienne, et la réplication persistante et l'intégration du génome hôte, bien qu'une possibilité, demeure un faible risque $(9,10)$. De plus, la Food and Drug Administration des États-Unis a recommandé qu'il n'est pas nécessaire de mettre fin à une étude si l'ADN plasmide reste inférieur à 30000 copies par $\mu \mathrm{g}$ d'ADN hôte dans les tissus hôtes (11).

Les vaccins d'ADN contre le coronavirus du syndrome respiratoire du Moyen-Orient (MERS-CoV) ont donné des résultats prometteurs dans les essais précliniques et sont ensuite passés à des essais cliniques de phase I. Toutefois, les vaccins n'ont pas progressé davantage, principalement parce que cette maladie est caractérisée par une épidémiologie changeante, ce qui signifie qu'au moment où les essais cliniques ont commencé pour le vaccin-candidat, l'incidence de la maladie avait considérablement diminué, ce qui présentait un obstacle potentiel à l'efficacité des essais (12). En 2016, pendant la crise du virus Zika, une étude préclinique menée chez des primates non humains à l'aide d'un vaccin contenant des structures d'ADN exprimant la protéine précurseur de la membrane et de l'enveloppe du virus a démontré la corrélation entre les niveaux d'anticorps et la protection. Avec ce succès, une étude clinique de phase I a été entreprise et les résultats préliminaires ont montré que le vaccin était sûr. Il a induit des anticorps neutralisants chez $62 \%$ des participants (13). Fait important, cette initiative a été entreprise peu après le décodage des séquences d'ADN des antigènes viraux, ce qui indique la vitesse à laquelle les vaccins d'ADN peuvent être produits.

Au printemps 2009, alors que le nouveau virus influenza H1N1 est devenu une pandémie mondiale, un essai clinique de phase I a été entrepris. En août 2009, une approche fondée sur l'ADN a été mise au point pour coder la protéine d'hémagglutinine A/California/04/2009 (H1N1pdm09). Bien que le vaccin contre l'ADN ait pu générer des niveaux d'anticorps contre l'inhibition de l'hémagglutination chez seulement $30 \%$ des sujets, les niveaux ont été portés à $72 \%$ dans les quatre semaines suivant la stimulation au moyen d'un vaccin antigrippal conventionnel homologué. Ces données suggèrent que le virus peut être contrôlé en utilisant l'ADN comme agent d'amorçage initial, suivi d'une stimulation par un vaccin conventionnel (14). II a été difficile de développer un vaccin contre le $\mathrm{VIH}$ en raison de la nature changeante du virus. Sur les six essais d'efficacité du vaccin contre le VIH-1 menés à ce jour, un seul (le RV144) s'est bien déroulé et est entré dans l'essai d'efficacité de la phase III. La mise au point de ce vaccin a été réalisée après plusieurs ratés en adoptant une strate d'amorçage avec des concepts d'ADN exprimant la variante $C$ gp120 et la variante B gp41, des protéines GAP et de protéase et en le stimulant avec le complexe protéique bivalent C gp120 du virus (15).

Le vaccin d'ARN (utilisant l'ARN messager [ARNm]) semble avoir certains avantages par rapport à ses homologues de vecteurs viraux et d'ADN. Comme l'ARNm n'interagit pas avec l'ADN de la cellule hôte, les vaccins d'ARNm ne présentent aucun risque d'intégration au génome de l'hôte. Par ailleurs, les vaccins d'ARNm ont une structure vectorielle simple contenant un cadre de lecture ouvert codant l'antigène cible comportant des gènes régulateurs spécifiques et ne sont donc pas capables d'induire 
une immunité anti-vectorielle (16). À I'heure actuelle, les vaccins contre le virus Zika, le chikungunya et certaines souches du virus de l'influenza font l'objet d'essais cliniques de phase I (11).

\section{Enjeux propres au coronavirus}

Les coronavirus sont des virus d'ARN à détection positive à simple brin. Ces virus sont de quatre genres (coronavirus alpha, bêta, gamma et delta); le SRAS-CoV-2 est un coronavirus bêta. Il se compose de quatre protéines structurales, à savoir un spicule, une enveloppe, une membrane et une nucléocapside, qui seraient impliqués dans l'invasion des cellules hôtes. Bien que des études soient toujours en cours pour mieux comprendre la biologie du SRAS-CoV-2, un éventail de vaccins-candidats ont commencé des essais cliniques et certains ont déjà été approuvés pour utilisation dans le monde entier. Les vaccins mis au point par Pfizer, AstraZeneca et Moderna se sont révélés efficaces dans une proportion de $90 \%$, et il est intéressant de noter qu'il s'agit de vaccins d'acide nucléique. En dépit de l'utilisation répandue de ces vaccins, il reste encore à répondre à certaines questions critiques, à savoir 1) les anticorps neutralisants et la réponse des lymphocytes $T$ spécifiques du SRAS-CoV-2 sont-ils suffisants pour prévenir la maladie et sa propagation ultérieure; 2) combien de temps dure l'immunité protectrice après l'infection ou la vaccination; 3) quels sont les facteurs responsables de la réaction immunitaire déréglée chez les patients qui ont des symptômes graves; et 4) le vaccin cause-t-il des réactions indésirables graves. Jusqu'à maintenant, aucun des vaccins contre la COVID-19 approuvée n'a soulevé de graves préoccupations en matière de sécurité; cependant, il reste des questions au sujet de leur sécurité relativement à leur utilisation à long terme et à leur efficacité contre les variants du SRAS-CoV-2. Habituellement, lorsqu'un vaccin est approuvé pour utilisation par le grand public, il fait l'objet d'évaluations d'innocuité rigoureuses afin de détecter les problèmes en effectuant des tests chez des dizaines de milliers de participants à l'étude, soit des études qui s'étendent sur plusieurs années. Apparemment, ce n'est pas ce qui s'est produit avec les vaccins contre la COVID-19; ces vaccins ont fait l'objet d'essais cliniques auprès de petits échantillons de participants et, en moins d'un an, leur utilisation chez les humains a été approuvée. Cette approche a été utilisée car nous étions au beau milieu d'une pandémie mondiale et qu'il était urgent de contrôler le virus, ce qui a entraîné le transfert de certains problèmes de sécurité et d'efficacité à régler principalement au moyen d'études postcommercialisation.

À l'avenir, que ce soit avec une modification des vaccins contre la COVID-19 actuellement approuvés ou avec un nouveau vaccin contre la COVID-19, il sera prudent de tenir compte des défis de développement expérimentés par d'autres vaccins viraux dans la mise au point des vaccins contre la COVID-19. II vaudrait la peine d'explorer une approche à multiples facettes, comme la stratégie de primo-immunisation qui a été utilisée pour les vaccins contre l'influenza et le $\mathrm{VIH}$ ou les orientations dérivées des études précliniques. Par exemple, dans une étude préclinique récente (17), six conceptions d'ADN exprimant différentes formes de protéines de spicule du SRAS-CoV-2 ont été utilisées pour vacciner des macaques rhésus. Les macaques présentaient des réponses immunitaires humorales et cellulaires et une réduction importante de la charge virale à la suite de la vaccination contre le SRAS-CoV-2. Bien que la taille de l'échantillon était petite $(n=4)$ pour chacun des groupes de vaccins-candidats, l'étude a laissé croire que la neutralisation des anticorps et le dépôt d'un complément dépendant des anticorps pourraient être des points de repère utiles à étudier pendant la mise au point d'un vaccin contre le SRAS-CoV-2.

\section{Conclusion}

Le temps est un facteur crucial dans la lutte contre les pandémies, mais l'efficacité et l'innocuité de tout vaccin sont également fondamentales. Lors de la conception d'un vaccin contre l'infection virale, il est particulièrement essentiel d'examiner quelles approches ont fonctionné et lesquelles n'ont pas fonctionné avec d'autres vaccins viraux.

\section{Déclaration de l'auteur}

R. C. a conçu et rédigé l'article

\section{Intérêts concurrents}

Aucun.

\section{Financement}

Aucun.

\section{Références}

1. Heath Kelly. The classical definition of a pandemic is not elusive. Bulletin of the World Health Organization 2011;89:540-541. DOI

2. Johnson NP, Mueller J. Updating the accounts: global mortality of the 1918-1920 "Spanish" influenza pandemic. Bull Hist Med 2002;76(1):105-15. DOI PubMed

3. Richardson JS, Dekker JD, Croyle MA, Kobinger GP. Recent advances in Ebolavirus vaccine development. Hum Vaccin 2010 Jun;6(6):439-49. DOI PubMed

4. Pronker ES, Weenen TC, Commandeur H, Claassen EH, Osterhaus AD. Risk in vaccine research and development quantified. PLoS One 2013;8(3):e57755. DOI PubMed 
5. Regules JA, Beigel JH, Paolino KM, Voell J, Castellano AR, Hu Z, Muñoz P, Moon JE, Ruck RC, Bennett JW, Twomey PS, Gutiérrez RL, Remich SA, Hack HR, Wisniewski ML, Josleyn MD, Kwilas SA, Van Deusen N, Mbaya OT, Zhou Y, Stanley DA, Jing W, Smith KS, Shi M, Ledgerwood JE, Graham BS, Sullivan NJ, Jagodzinski LL, Peel SA, Alimonti JB, Hooper JW, Silvera PM, Martin BK, Monath TP, Ramsey WJ, Link CJ, Lane HC, Michael NL, Davey RT Jr, Thomas SJ; rVSV $\triangle$ G-ZEBOV-GP Study Group. A recombinant vesicular stomatitis virus Ebola vaccine. $\mathrm{N}$ Engl J Med 2017;376(4):330-41. DOI PubMed

6. Baldo A, van den Akker E, Bergmans HE, Lim F, Pauwels K. General considerations on the biosafety of virus-derived vectors used in gene therapy and vaccination. Curr Gene Ther 2013;13(6):385-94. DOI PubMed

7. Prazeres DM, Monteiro GA. Plasmid Biopharmaceuticals. Microbiol Spectr 2014;2(6):1-18. DOI PubMed

8. Maslow JN. Vaccines for emerging infectious diseases: lessons from MERS coronavirus and Zika virus. Hum Vaccin Immunother 2017;13(12):2918-30. DOI PubMed

9. Hu Y, Li W, Gao T, Cui Y, Jin Y, Li P, Ma Q, Liu X, Cao C. The severe acute respiratory syndrome coronavirus nucleocapsid inhibits type I interferon production by interfering with TRIM25-mediated RIG-I ubiquitination. J Virol. 2017;91:e02143-16. DOI PubMed

10. Huang $C$, Wang $Y$, Li X, Ren $L$, Zhao J, Hu Y, Zhang L, Fan G, Xu J, Gu X, Cheng Z, Yu T, Xia J, Wei Y, Wu W, Xie X, Yin W, Li H, Liu M, Xiao Y, Gao H, Guo L, Xie J, Wang G, Jiang R, Gao Z, Jin Q, Wang J, Cao B. Clinical features of patients infected with 2019 novel coronavirus in Wuhan, China. Lancet 2020;395(10223):497-506. DOI PubMed

11. Sariol A, Perlman S. Lessons for COVID-19 Immunity from other coronavirus infections. Immunity 2020;53(2):248-63. DOI PubMed

12. Diamond MS, Pierson TC. The challenges of vaccine development against a new virus during a pandemic. Cell Host Microbe 2020;27(5):699-703. DOl PubMed
13. Kiambi S, Corman VM, Sitawa R, Githinji J, Ngoci J, Ozomata AS, Gardner E, von Dobschuetz S, Morzaria S, Kimutai J, Schroeder S, Njagi O, Simpkin P, Rugalema G, Tadesse Z, Lubroth J, Makonnen Y, Drosten C, Müller MA, Fasina FO. Detection of distinct MERS-Coronavirus strains in dromedary camels from Kenya, 2017. Emerg Microbes Infect 2018;7(1):1-4. DOI PubMed

14. Josset L, Menachery VD, Gralinski LE, Agnihothram S, Sova P, Carter VS, Yount BL, Graham RL, Baric RS, Katze MG. Cell host response to infection with novel human coronavirus EMC predicts potential antivirals and important differences with SARS coronavirus. MBio 2013;4(3):e00165-13. DOI PubMed

15. Hsu DC, O'Connell RJ. Progress in HIV vaccine development. Hum Vaccin Immunother 2017;13(5):1018-30. DOI PubMed

16. Shaman J and Glanti M. Direct measurement of rates of asymptomatic infection and clinical care-seeking for seasonal coronavirus, MedRxiv. 2020;2020.01.30.20019612. https://www.medrxiv.org/content/10.1101/2020.01.30.2001 9612v1.full.pdf

17. Yu J, Tostanoski LH, Peter L, Mercado NB, McMahan K, Mahrokhian SH, Nkolola JP, Liu J, Li Z, Chandrashekar A, Martinez DR, Loos C, Atyeo C, Fischinger S, Burke JS, Slein MD, Chen Y, Zuiani A, Lelis FJ, Travers M, Habibi S, Pessaint L, Van Ry A, Blade K, Brown R, Cook A, Finneyfrock B, Dodson A, Teow E, Velasco J, Zahn R, Wegmann F, Bondzie EA, Dagotto G, Gebre MS, He X, Jacob-Dolan C, Kirilova M, Kordana N, Lin Z, Maxfield LF, Nampanya F, Nityanandam R, Ventura JD, Wan H, Cai Y, Chen B, Schmidt AG, Wesemann DR, Baric RS, Alter G, Andersen $\mathrm{H}$, Lewis MG, Barouch DH. DNA vaccine protection against SARS-CoV-2 in rhesus macaques. Science 2020;369(6505):806-11. DOI PubMed 